\title{
Why Doesn’t Adversity Make Everyone Stronger? Mediators of the Relationship Between Trait Emotion and Resilience
}

\author{
George K., Clarke J. \\ University of York, York, United Kingdom
}

\begin{abstract}
High levels of negative trait emotion have been shown to increase the likelihood that individuals are unable to "bounce back" from adversity. Some researchers attribute this to the intense negative emotional reactions associated with negative trait emotion. On the other hand, however, others argue that negative emotions are necessary for developing higher levels of resilience. Therefore, the question remains: What causes individuals with high levels of trait emotion to adapt poorly to adversity? This paper claimed that rumination may play an important role. Rumination has consistently been implicated as having a negative impact on resilience. Therefore, the research compared the mediating influence of negative emotions and rumination on the relationship between trait anger, trait depression, trait anxiety, and resilience. One hundred and six students from the University of York filled in measures assessing their levels of trait emotion, rumination, and resilience. They also rated their emotional reactions to several videos. Analyses indicated that higher levels of rumination partially mediated the relationship between all trait emotions and resilience. However, the emotional reactions did not have any mediating effect. The findings highlighted the negative impact of prolonged rumination on resilience. Future research should explore other cognitive mechanisms which may also account for the lower levels of resilience associated with trait emotion.
\end{abstract}

Keywords: adversity, trait emotion, emotional reaction, rumination, resilience

\section{Introduction}

Resilience refers to the capacity for individuals to emerge from negative experiences without developing psychopathology (Masten, 2001). Research has recently highlighted the importance of positive emotions to promoting these adaptive responses. For instance, the broaden-and-build theory posits that positive emotions broaden one's thought-action repertoire, increasing the range of coping behaviours that the person considers when handling the negative event. In support of this theory, positive emotions have been found to enable resilient individuals to "bounce back" quickly and efficiently from a range of negative experiences (Fredrickson, Tugade, Waugh, \& Larkin, 2003; Tugade \& Fredrickson, 2004; Fredrickson \& Branigan, 2005; Ong, Bergeman, Bisconti, \& Wallace, 2006). It has also been linked to the increased use of resilient coping behaviours (Folkman \& Moskowitz, 2003), higher levels of self-esteem (Benetti \& Kambourpoulos, 2006), and an improved capacity to engage in creative problem-solving (Isen, Daubman, \& Nowicki, 1987; Rowe, Hirsch, \& Anderson, 2007). As such, treatments have emerged focus on promoting positive emotions, for instance, through learned

George K., B.Sc. (Hons), M.Sc. (Merit), Ph.D. researcher, Psychology Department, University of York.

Clarke J., C.Psychol., C.Sci., AFBPsS., EuroPsy., HPC Registered Forensic Psychologist, Psychology Department, University of York. 
optimism (Frederickson, 2000).

On the other hand, negative emotions have been criticised as one of the major causes for difficulty in adapting to adversity. For example, the broaden-and-build theory posits that negative emotions constrict the thought-action repertoire, essentially causing the individual to revert to automatic instinctive reactions, such as attacking when angry. In support of this, findings have revealed that negative emotions hinder processing (Baron, Inman, Kao, \& Logan, 1992) and promote false memories (Brainerd, Stein, Silveira, Rohenkohl, \& Reyna, 2008). Additionally, high levels of negative trait emotion (the tendency to consistently experience intense, frequent, and prolonged negative emotions across time and situations, even in the absence of aversive stimuli) have been linked to poor adaptation to a variety of negative events. For instance, in response to intense trauma, such as victimization, higher levels of trait depression have been found to promote delinquency (Manasse \& Ganem, 2009). High trait anxiety has been shown to negatively impact individuals' levels of self-esteem (Benetti \& Kambouropoulos, 2006). Self-esteem has been described as a fundamental to resilience (Rutter, 1985; Fine, 1991). Furthermore, trait anger has been associated with juvenile delinquency, misuse of alcohol, and aggressive and risky behaviour while driving and more "close calls" and minor accidents (Deffenbacher, 1992; Cornell, Peterson, \& Richards, 1999; Deffenbacher, Huff, Lynch, Oetting, \& Natalie, 2000).

The emotional reactions associated with trait emotion have been highlighted as the main cause for these negative responses. For instance, trait anxiety is defined as the relatively stable tendency to perceive a variety of events as dangerous or threatening in nature. Individuals high in trait anxiety thus experience intense state anxiety, accompanied by physiological arousal and a range of negative emotions such as tension and apprehension, to such perceived threats (Spielberger \& Sydeman, 1994). As a result, its impact on behaviour differs from that of other trait emotions. For instance, Fox and Spector (1999) showed differences in the influence of trait anxiety and trait anger on affective responses and counterproductive behaviours to negative job experiences such as depending on others who are not well trained. The researchers found that individuals high on trait anxiety, not trait anger, were more likely to report feelings of job frustration, assessed with items such as "trying to get this job done is a very frustrating experience". Analyses using Analyses using LISREL (Linear Structural Relations) 8 indicated that such feelings of frustration mediated the relationship between trait anxiety and behavioural reactions including starting an argument with someone at work. On the other hand, the study revealed that trait anger was directly related to these counterproductive behaviours. Although the results are constrained by the fact that the measure of affective response failed to provide sufficient details on the nature of the emotional reaction, it hints at the different implications that the various negative emotions may have on individuals' levels of resilience.

However, in more recent times, researchers assert that negative emotions have significant benefits to promoting higher levels of resilience. They claimed that such emotions enable the individual to make meaning of their experiences by triggering the cognitive processing necessary for understanding the adverse event (Horowitz, 1975; Tedeschi \& Calhoun, 2004). In support of this, the literature has shown that resilient individuals do not experience significantly less negative emotions when handling adversity (Tugade \& Frederickson, 2004; Ong et al., 2006). In addition, findings suggest that emotional outcomes mainly influence individuals' immediate responses to the situation. For instance, in terms of trait anger, the intense angry feelings mainly account for instances of reactive aggression, that is, impulsive hostile responses to the perceived threat or provocation (Dodge \& Coie, 1987). On the other hand, however, an individual's level of 
resilience does not rely solely on their reactions within the "chemistry of the moment” (Rutter, 2007, p. 205). Many studies show that in spite of initial maladaptive responses, the majority of individuals are capable of eventually developing high levels of resilience and thus exhibiting more adaptive behaviour (Flach, 1988; Fine, 1991; Richardson, 2002; Rutter, 2007). For instance, the resilience levels of clinical patients increased following exposure to treatment (Connor \& Davidson. 2003). These findings thus suggest that the lower levels of resilience associated with negative trait emotion cannot be fully attributed to its associated predisposition toward frequent, intense, and enduring negative emotional responses to adverse experiences.

Research has instead suggested that cognitive processes may be influential in cases of high negative trait emotion. For instance, Tafrate, Kassinove, and Dundin (2002) evaluated the characteristics of anger episodes of individuals high on trait anger within the community. As anticipated, they found a strong relationship between trait anger and experiences of anger: 53\% of the sample became intensely angry "a few days a week" (ratings were 90 or above on a scale whose maximum is 100) with episodes typically lasting "more than a day" for persons high on trait anger. In addition, the findings revealed the maladaptive responses to negative experiences typically associated with high trait anger. For example, the majority of high trait anger respondents were more likely to yell, scream, argue, threaten, and make sarcastic and abusive remarks. In addition, physical aggression was three times more likely to occur in cases of high trait anger than low trait anger participants. Within this study in particular, however, the researchers highlighted the presence of cognitive biases. High trait anger participants were four times more likely to admit that their perceptions of the anger-provoking event were "distorted". They also reported significantly higher rates of catastrophizing, low frustration tolerance, and negative self-ratings. However, the role these biases played in generating the aggressive behaviour was not directly assessed. Therefore, the impact of cognition in cases of negative trait emotion warrants further investigation.

Of the many cognitive processes explored in the literature, rumination has been highlighted as the most influential in determining individuals' levels of resilience. Rumination is characterized by repetitive, intrusive, and negative cognitions and a range of other physiological and emotional symptoms. Morrow and Nolen-Hoeksema (1990) and Michael and Snyder (2005, p. 436) described rumination as a continuous focus on negative emotions and what these emotions mean "without getting any closer to finding a solution that lessens these feelings". They argued that rumination represents a distressing but necessary and temporary phase of the meaning making process. However, individuals can "get stuck" in the stage of rumination, and thus become unable to find meaning in the experience. Without meaning, resilience is not developed (Horowitz, 1975; Janoff-Bulman, 1992; Greenberg, 1995; Tedeschi \& Calhoun, 2004). For instance, among American males positive with human immuno-deficiency virus, Bower, Kemeny, Taylor, and Fahey (1998) found that individuals who were still engaged in the initial stages of cognitive processing were not as likely to report adaptive outcomes, whereas among participants who survived colorectal cancer, Salsman, Segerstrom, Brechting, Carlson, and Andrykowski (2009) found that such processing increased the prevalence of post-traumatic stress disorder. In addition, rumination has been shown to "dampen" any positive emotions that may be triggered (Feldman, Joormann, \& Johnson, 2008). Research has hinted that negative trait emotion may be associated with a tendency to engage in prolonged periods of rumination following adversity. For example, as aforementioned, Tafrate, Kassinove, and Dundin (2002) revealed that high trait anger individuals reported experiencing ruminative and irrational thinking. Rumination may thus provide a significant explanation for the relationship between negative trait emotion and resilience. 


\section{Current Study}

The present study was novel for two main reasons. Primarily, it sought to explore negative trait emotion while avoiding the methodological flaws which exist in the current trait emotion literature. Previous empirical work assessing negative trait emotions mainly incorporates artificial stimuli such as words (Mogg, Bradley, \& Halowell, 1994; Parrot, Zeichner, \& Evces, 2005) and faces (Van Honk et al., 2001). Such an emphasis on laboratory-based research may provide results which have limited ecological validity. For instance, Mogg et al. (1994) showed that under lab-induced anxiety, individuals high on trait anxiety were not significantly more likely to exhibit attentional biases. On the other hand, under naturally induced anxious conditions, significant attentional biases emerged. In addition, attempts to analyse individuals' real world experiences usually assess events associated with the specific negative trait emotion being investigated. For example, Tafrate et al. (1989) only assessed individuals on their anger episodes in their exploration of the impact of trait anger on everyday life. Therefore, certain gaps remain. For example, research still needs to determine how individuals high on trait anger will react to potentially anxiety-provoking stimuli. Or even more important, how do each of the traits and their associated emotional reactions influence resilience after exposure to the same stimuli. This can be performed using videos, as was done by Hazebroek et al. (2001) in their analysis of trait anger. A range of videos displaying everyday experiences ranging in severity were utilized in the current study.

Secondly, this research compared the roles of emotion and rumination on the relationship between negative trait emotion and resilience-a topic not previously dealt with in the literature. It was hypothesized that negative trait emotion will be negatively correlated with resilience but positively correlated with faster and more intense emotion-congruent reactions to video stimuli and rumination scores. Negative correlations were also hypothesized between rumination scores and resilience; and emotional responses and resilience. Significant mediating relationships were anticipated in accordance with the mediation models presented in Figure 1.

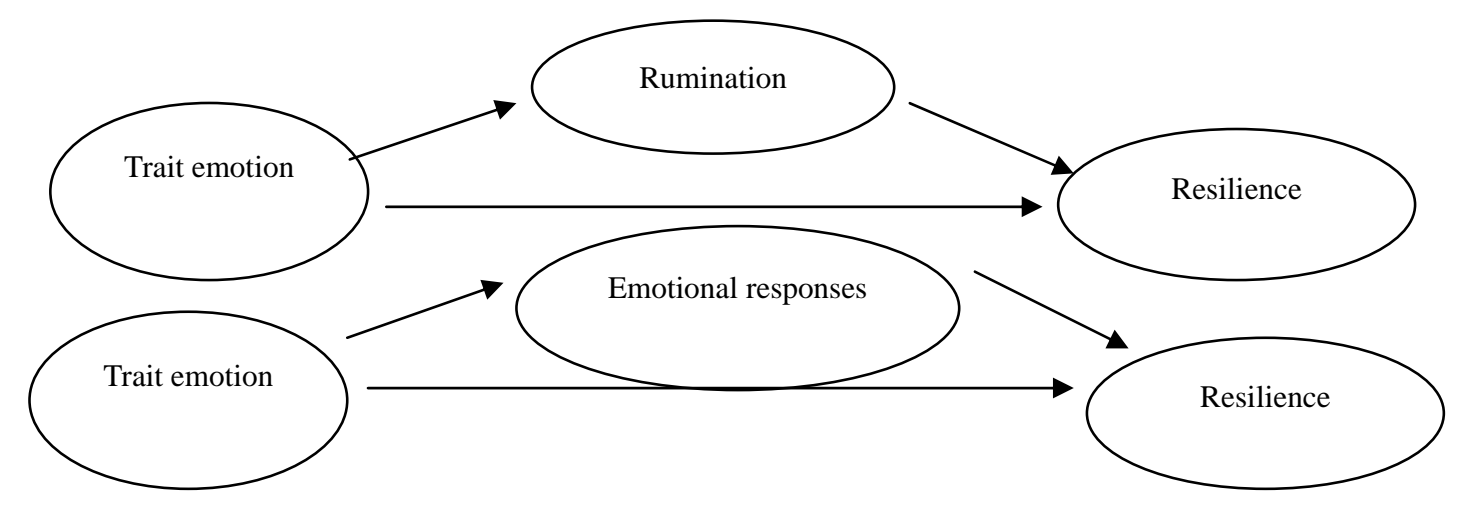

Figure 1. Graphic representation of the mediation models.

\section{Materials and Methods}

\section{Participants}

One hundred and six staff and students from the University of York (53 males and 53 females) participated in the present study in return for a small financial compensation or course credit. The sample was collected using online and poster advertising around the University of York campus. Their mean age was 22.63 $(S D$ (standard deviations) $=4.28)$. The sample included British (45.3\%), European (17\%), Asian (12.3\%), Chinese (19.8\%), American (0.9\%), African (2.8\%), Brazilian (0.9\%), and Hispanic (0.9\%) individuals. 


\section{Measures}

(1) Rumination scores were obtained using the rehearsal subscale of the ECQ (Emotion Control Questionnaire) (Roger \& Najarian, 1989). It uses 14 dichotomous (true/false) items (e.g., I remember things that upset me or make me angry for a long time afterwards) to assess the individual's tendency to ruminate over emotionally upsetting events. Higher scores reflect a greater tendency to engage in rumination with a total possible score of 14 . Research within a similar undergraduate population documented a large alpha coefficient of 0.86;

(2) Resilience was evaluated with the CD-RISC (Connor-Davidson resilience scale) (Connor \& Davidson, 2003), which is a 25-item questionnaire assessing successful stress-coping abilities. Items such as "I can deal with whatever comes my way" are rated on a 5-point scale with $1=$ "Not at all typical of me" and $5=$ "True nearly all the time”. Scores are summed up to determine resilience levels, with a possible range of $0-100$. Higher scores represent higher levels of resilience. Within the sample of 1,644 individuals of the general population in which it was initially tested, an alpha coefficient of 0.89 was reported;

(3) Trait anger was measured using the trait subscale of the STAXI-II (State-Trait Anger Expression Inventory-2) (Spielberger, 1999). It consists of 10 items assessing the dispositional tendency of individuals to react to experiences with intense feelings of anger. For example, it asks participants whether they are generally quick-tempered on a scale of 1 (“Almost never”) to 4 (“Almost always”). After recoding reversed items, a total trait anger score can be derived by summing eight of the 10 questions. Although the subscale of trait anger (reactivity) emerged with alphas of 0.76 and 0.73 for females and males respectively, the trait anger scale has demonstrated good internal consistency in non-clinical adult samples (alphas $=0.84-0.86$ );

(4) Trait anxiety was assessed using the trait anxiety subscale of the STAI (State-Trait Anxiety Inventory) (Spielberger, Jacobs, Russell, \& Crane, 1983). This measures dispositional anxiety and comprises 20 items evaluated on a scale of 1 (“Almost never") to 4 (“Almost always”). It includes items such as "I feel nervous and restless”. Levels of trait anxiety are calculated by recoding reverse scored items and adding all scores. Spielberger et al. (1983) reported the median alpha coefficient for large independent samples of college undergraduates, military recruits, and working adults as 0.90 ;

(5) Trait depression was indexed by the trait depression subscale of the STPI-TD (State-Trait Personality Inventory) (Spielberger, Reheiser, \& Sydeman, 1995). It includes 10 questions measuring individual's dispositional tendency to react to experiences with intense feelings of depression. Items such as "I feel hopeless" are rated on a scale of 1 (“Almost never") to 4 (“Almost always”). Several reverse scored items are included. Trait-depression scores are attained by totalling all items. The scale has strong internal consistency producing alpha coefficients above 0.80 for both genders;

(6) Emotional responses were assessed by asking participants to rate 10-second clips as fast as possible. There were 24 clips in addition to three practice videos depicting such events as cats fighting, a woman having difficulty standing after slipping on ice, and children eating. These were chosen from an initial sample of 37 silent clips standardized by 10 participants who rated them in terms of how angry, sad, and anxious they would feel within the various scenes depicted.

Before viewing the videos, participants were told to imagine they were the main character in the scene or related to the main character in some way, for example, as a passer-by or a friend. The video clip was then played. The order of videos was randomized. After each clip, participants were presented with three consecutive scales on which they rated how angry, anxious, and sad they would feel under the stated 
circumstances. The scales ranged from 1 ("Not at all”) to 6 ("Extremely") and were also presented in a random order. Higher ratings signified more intense emotional reactions. Henceforth, anger ratings will be referred to as angrat; anxiety ratings will be called anxrat and depression ratings will be deprat.

\section{Procedure}

Following informed consent, all participants filled out a demographic questionnaire where they reported their age, ethnicity, gender, and whether they spoke English as a first language. The experiment involved two major components: a series of self-report questionnaires assessing rumination, resilience, trait anger, trait anxiety and trait depression, and ratings of videos to determine emotional responses. These two conditions were counterbalanced.

Preliminary analyses revealed that scores on several of the measures were significantly positively skewed. This is anticipated based on the non-clinical nature of the sample (Spielberger et al., 1983). Logarithmic transformations successfully removed the skews on angrat, anxrat, trait depression, and response times for anxiety and sadness. However, trait anger and rumination scores remained skewed, even after the removal of outliers. Therefore, bootstrapping was used to assess the hypothesized mediating relationships (trait emotion $\rightarrow$ rumination scores $\rightarrow$ resilience) and (trait emotion $\rightarrow$ emotion ratings $\rightarrow$ resilience). Bootstrapping is a non-parametric procedure used to determine mediating effects. It generates a 95\% confidence interval which, if it includes zero, is said to depict a partial mediation (Preacher \& Hayes, 2008).

\section{Results}

\section{Descriptive Statistics}

Means, $S D$, and ranges are reported in Table 1 . The means of the current sample were generally comparable to prior research in similar undergraduate populations (Roger \& Najarian, 1989; Spielberger et al., 1983; Spielberger et al., 1995; Spielberger, 1999; Litman, Collins, \& Spielberger, 2005; Bitsika, Sharpley, \& Peters, 2010; Culhane \& Morera, 2010).

Table 1

Means, SD, and Ranges for the Predictor and Outcome Variables

\begin{tabular}{lccc}
\hline Variable & Mean & $S D$ & Range \\
\hline Trait anger & 19.03 & 4.17 & 18.00 \\
Trait anxiety & 40.59 & 9.05 & 46.00 \\
Trait depression & 18.71 & 4.58 & 22.00 \\
Angrat & 2.38 & 0.64 & 3.41 \\
Anxrat & 3.14 & 0.80 & 3.46 \\
Deprat & 2.42 & 0.64 & 3.79 \\
Speed anger ratings & $1,818.10 \mathrm{~ms}$ & $740.38 \mathrm{~ms}$ & $4,467.71$ \\
Speed anxiety ratings & $1,697.08 \mathrm{~ms}$ & $722.98 \mathrm{~ms}$ & $3,972.45$ \\
Speed depression ratings & $2,409.32 \mathrm{~ms}$ & $1,044.47 \mathrm{~ms}$ & $5,030.34$ \\
Rumination & 5.94 & 3.00 & 13.00 \\
Resilience & 69.67 & 10.53 & 58.00 \\
\hline
\end{tabular}

\section{Preliminary Analyses}

Independent samples $t$-tests did not reveal significant gender differences on any of the variables. Individuals who spoke English as a first language responded significantly faster for all emotions: anger, $t_{(104)}=$ 3.03, $p<0.01$; anxiety, $t_{(104)}=2.03, p<0.05$; and sadness, $t_{(104)}=2.78, p<0.01$. This was also reflected in 
White British students giving significantly faster depression responses than Chinese students, $t_{(77)}=-2.20, p<$ 0.05. However, as the speed data did not correlate significantly with any of the other variables, it was not included in any further analyses.

\section{Correlations}

Table 2 shows the correlations between negative trait emotion, rumination, emotion ratings, and resilience. As anticipated, scores on each trait emotion were significantly positively correlated with emotion ratings and rumination scores as well as negatively correlated with scores on the resilience measure. Rumination scores were also significantly negatively correlated with resilience. However, there was no relationship between ratings and resilience scores.

Table 2

Correlations Between Trait Emotion, Emotion Ratings, Rumination Scores, and Resilience

\begin{tabular}{|c|c|c|c|c|c|c|c|c|}
\hline Variable & 1 & 2 & 3 & 4 & 5 & 6 & 7 & 8 \\
\hline (1) Trait anger & & $0.29^{* *}$ & $0.20^{* * *}$ & $0.26^{* *}$ & 0.05 & -0.04 & $0.33^{* *}$ & $-0.21^{*}$ \\
\hline (2) Trait anxiety & & & $0.45^{* *}$ & 0.14 & $0.22^{*}$ & -0.02 & $0.29^{* *}$ & $-0.36^{* *}$ \\
\hline (3) Trait depression & & & & 0.13 & -0.08 & $0.39^{* *}$ & $0.37^{* *}$ & $-0.24^{* *}$ \\
\hline (4) Angrat & & & & & 0.06 & $0.30^{* *}$ & $0.20^{* *}$ & -0.02 \\
\hline (5) Anxrat & & & & & & -0.10 & -0.03 & -0.10 \\
\hline (6) Deprat & & & & & & & $0.20^{* *}$ & -0.08 \\
\hline (7) Rumination & & & & & & & & $-0.32^{* *}$ \\
\hline (8) Resilience & & & & & & & & \\
\hline
\end{tabular}
Notes. ${ }^{*} p<0.05 ;{ }^{* *} p<0.01$.

\section{Mediation Analyses}

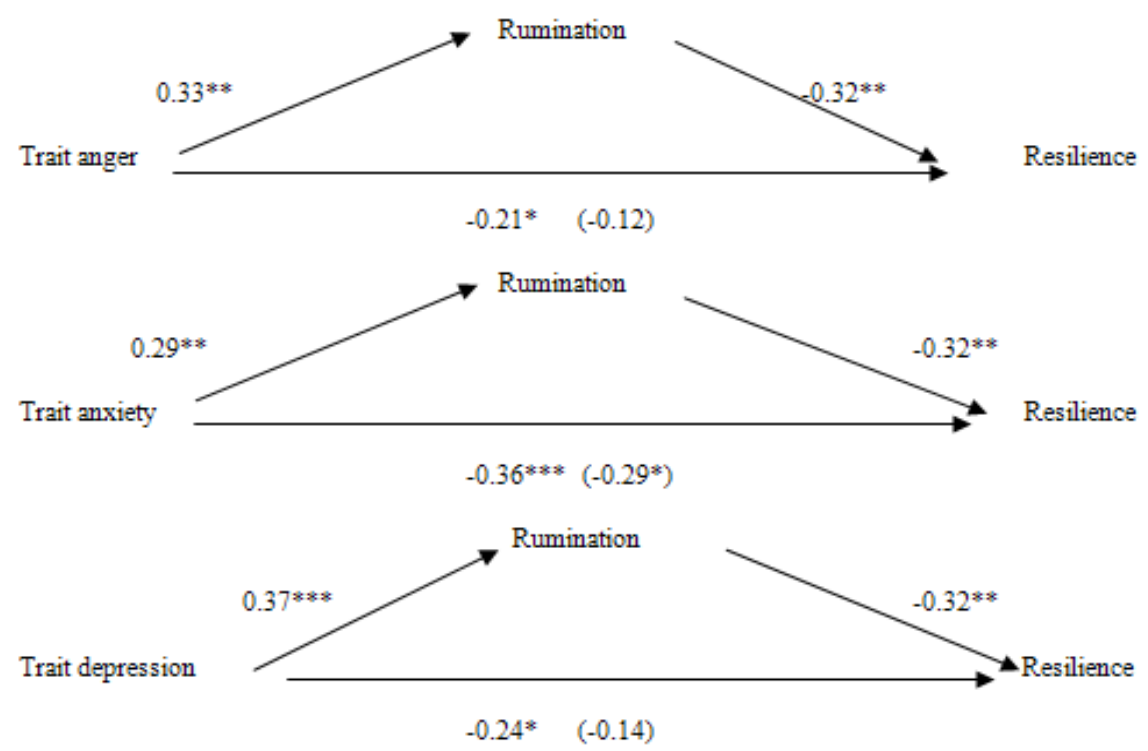

Figure 2. Results of the mediating analyses.

The results are presented in Figure 2 where standardized regression coefficients are reported. In the first mediational analysis, resilience was entered as the dependent variable, trait anger was the independent variable and rumination and anger ratings were the two potential mediators. The true confidence intervals for rumination 
and anger ratings respectively are -1.6 and -0.33 and -2.27 and 4.15 at $p<0.01$. As zero was not included in the interval for rumination, it can be concluded to have partially mediated the relationship between trait anger and resilience.

In the second analysis, resilience was the dependent variable, trait anxiety was the independent variable and rumination and anxiety ratings were entered as two mediators. The confidence intervals for rumination and anxiety ratings respectively are -1.41 and -0.26 and -4.16 and 3.16 at $p<0.01$. The results show that rumination partially mediates the relationship between trait anxiety and resilience, as its interval did not include zero.

In the third analysis, resilience was the dependent variable, trait depression was the independent variable and rumination and depression ratings were the two potential mediators. The confidence intervals for rumination and depression anxiety are -1.51 and -0.36 and -3.40 and 4.43 at $p<0.01$. It can be concluded that rumination partially mediates the relationship between trait depression and resilience as zero was not included in its interval.

According to Raes (2010), the analyses were performed again entering the other trait emotions as extra covariates. The results remained largely unchanged. The confidence interval for rumination did not contain zero: -1.442 and -0.05 at $p<0.05$.

\section{Discussion}

In support of the hypothesis, higher scores on all negative trait emotions were significantly negatively correlated with resilience. This corroborates prior findings concerning the impact of the various negative trait emotions on adaptive outcomes following negative experiences (Moosbrugger \& Schermelleh-Engel, 1991; Fox \& Spector, 1999; Manasse \& Ganem, 2009). Although constrained by the use of correlations, which do not allow for causal analyses, the results substantiate the relevance of exploring resilience among individuals high on negative trait emotion.

As anticipated, levels of negative trait emotion were significantly positively correlated with emotion-congruent ratings. Higher levels of trait anger were related to more extreme anger ratings of the stimuli while increased trait anxiety and trait depression triggered more intense anxiety and sadness ratings respectively. The findings suggest that when individuals high on trait anger confront a negative experience, they would feel severe anger while individuals high on trait anxiety reported that they would feel extremely anxious if exposed to the same event. Therefore, the current study implies that each negative trait emotion is associated with distinct emotional reactions. This substantiates the results of prior research assessing the relationship between negative trait emotion and automatic emotional responses (MacLeod \& Rutherford, 1992; Parrot, Zeichner, \& Evces, 2005; Wilkowski \& Robinson, 2008). Furthermore, it adds to the existing literature as it sheds light on how each trait influences their emotional responses not only to the same stimuli but also in relation to real life experiences.

Contrary to the hypotheses, although emotion ratings were all negatively correlated with resilience, none of the relationships were significant. In addition, there were no significant mediating relationships between trait emotion, emotional reactions, and resilience. These findings suggest that although intense emotional reactions increase the likelihood that individuals will respond to negative experiences with maladaptive behaviours such as aggression (Wilkowski \& Robinson, 2008a), these initial responses in no way influence their capacity to eventually respond to similar adversities in an adaptive manner. This is also reflected in the literature. For instance, there are no significant differences in the experience of negative emotions following adversity among 
resilient and non-resilient individuals (Tugade \& Frederickson, 2004; Ong et al., 2006).

As anticipated, rumination scores were significantly positively correlated with all negative trait emotions and significantly negatively correlated with resilience. In addition, the current study showed that rumination mediated the relationship between negative trait emotion and resilience while the negative emotional reactions did not. These results support prior findings concerning the relationship between negative trait emotion and rumination (Roger \& Najarian, 1989; Tafrate et al., 2002). They also substantiated the harmful impact of prolonged rumination on resilience levels, as shown in previous research (Nolen-Hoeksema, 1991; Salsman et al., 2009). The current study thus corroborates the importance of cognitive mechanisms to understanding the relationship between trait emotion and resilience.

However, only partial mediations were found between trait emotion, rumination, and resilience. These findings suggest that other aspects of cognition may exist which are pertinent to explaining the relationship between trait emotion and resilience. The current study may thus be constrained by only incorporating a measure of rumination. Furthermore, the ECQ (Emotion Control Questionnaire) assessed only one aspect of the multidimensional rumination construct. Factor analyses have revealed three main dimensions (Siegle, Moore, \& Thase, 2004). These included a negative focus on distant past events; generally negatively valenced thought such as worry which focuses on oneself, one's life, and one's experiences; and reflective pondering or neutrally valenced consideration of oneself, one's life, and one's experiences. The ECQ was included in Siegle et al.'s (2004) study and emerged as an assessment of factor 2-generally negatively valenced thought. Therefore, further research is needed which assesses the influence of other dimensions of rumination and aspects of cognition on resilience.

The findings are also constrained by the resilience measure used. Empirical work has emphasized the complexity of the resilience construct. For instance, some researchers conceptualize resilience as a coping mechanism (Connor \& Davidson, 2003) while others emphasize social and emotional functioning (Campbell-Sills, Cohen, \& Stein, 2006). On the other hand, researchers frequently emphasize the need to utilize qualitative methods as maladaptive behaviours may for some populations signify resilience. For instance, Hunter and Chandler (1999) claimed that the current measures of resilience that exist do not allow for an understanding of the various ways in which resilience may manifest. They explored resilience among a sample of aggressive inner city adolescents within the United States and found that for them, resilience meant survival at all costs. In fact, individuals capable of emotional distancing and aggression were deemed the most resilient. Therefore, the current findings may be constrained by the use of the CD-RISC to assess resilience. Future research aimed at investigating resilience should incorporate several quantitative measures as well as qualitative research methods.

In addition, the speed ratings were not significantly correlated with any negative trait emotion. A significant relationship between negative trait emotion and faster reaction times has not consistently been found in the literature (see Rusting, 1998). Rusting (1998) attributed this to the absence of sufficiently emotional stimuli which would trigger the intense emotional reactions necessary to generate quicker responses. However, negative trait emotion is defined as a general dispositional tendency to experience intense negative emotions, even in the absence of aversive stimuli. Therefore, the emotional intensity of the videos used may not account for the current findings. A more pertinent explanation may lie in the use of video stimuli. Although Hazebroek, Howells, and Day (2001) previously utilized similar videos to assess the patterns in cognitive appraisal associated with trait anger, their study did not require participants to give their emotional reactions as promptly 
as is asked in the current research. Instead, a booklet was used for the undergraduates involved to make note of their appraisals and emotional responses. Therefore, the videos may have been too complicated to demand automatic responses. Further research is therefore still needed to determine whether negative trait emotion leads to speedier emotion congruent reactions.

The current study aimed to compare the mediating influence of negative emotional responses and rumination on the relationship between different negative trait emotions and resilience. The results substantiated the mediating influence of rumination, not negative emotional reactions. However, the limitations induced by the rumination and resilience measures utilized limit the current study. It is thus suggested that future research incorporate qualitative methods as well as a wider range of measures assessing rumination and resilience. The study nonetheless substantiates the influence of cognition on the relationship between negative trait emotion and resilience. More importantly, however, it hints at the need for further exploration into the various cognitive mechanisms which may explain the lower levels of resilience associated with high negative trait emotion. Only in this way can research answer why adversity fails to strengthen individuals high on trait emotion.

\section{References}

Almedom, A. M., \& Glandon, D. (2007). Resilience is not the absence of PTSD any more than health is the absence of disease. Journal of Loss and Trauma, 12, 127-143.

Baron, R. S., Inman, M., Kao, C., \& Logan, H. (1992). Emotion and superficial social processing. Motivation and Emotion, 16, 323-345.

Benetti, C., \& Kambouropoulos, N. (2006). Affect-regulated indirect effects of trait anxiety and trait resilience on self-esteem. Personal and Individual Differences, 41, 341-352.

Bitsika, V., Sharpley, C., \& Peters, K. (2010). How is resilience associated with anxiety and depression? Analysis of factor score interactions within a homogenous sample. German J. Psych., 13, 9-16.

Bower, J. E., Kemeny, M. E., Taylor, S. E., \& Fahey, J. L. (1998). Cognitive processing, discovery of meaning, CD4 decline, and AIDS-related mortality among bereaved HIV-seropositive men. Journal of Consulting and Clinical Psychology, 66(6), 979-986.

Brainerd, C. J., Stein, L. M., Silveira, R. A., Rohenkohl, G., \& Reyna, V. F. (2008). How does negative emotion cause false memories? Psychological Science, 19, 919-925.

Campbell-Sills, L., Cohan, S. L., \& Stein, M. B. (2006). Relationship of resilience to personality, coping, and psychiatric symptoms in young adults. Behaviour Research and Therapy, 44, 585-599.

Connor, K. M., \& Davidson, J. R. T. (2003). Development of a new resilience scale: The Connor-Davidson Resilience Scale (CD-RISC). Depression and Anxiety, 18, 76-82.

Cornell, D. G., Peterson, C. S., \& Richards, H. (1999). Anger as a predictor of aggression among incarcerated adolescents. Journal of Consulting and Clinical Psychology, 67(1), 108-115.

Culhane, S. E., \& Morera, O. F. (2010). Reliability and Validity of the Novaco Anger Scale and Provocation Inventory (NAS-PI) and State-Trait Anger Expression Inventory-2 (STAXI-2) in Hispanic and Non-Hispanic White student samples. Hispanic Journal of Behavioral Sciences, 32(4), 586-606.

Deffenbacher, J. L. (1992). Trait anger: Theory, findings, and implications. In C. D. Spielberger, \& J. N. Butcher (Eds.), Advances in personality assessment (Vol. 9, pp. 177-201). Hillsdale, N. J.: Lawrence Erlbaum Associates.

Deffenbacher, J. L., Huff, M. E., Lynch, R. S., Oetting, E. R., \& Natalie, F. (2000). Characteristics and treatment of high-anger drivers. Journal of Counseling Psychology, 47(1), 5-17.

Dodge, K. A., \& Coie, J. D. (1987). Social information-processing factors in reactive and proactive aggression in children's playgroups. Journal of Personality and Social Psychology, 53, 1146-1158.

Feldman, G., Joormann, J., \& Johnson, S. L. (2008). Responses to positive affect: A self-report measure of rumination and dampening. Cognitive Therapy and Research, 32, 507-525. 
Fine, S. B. (1991). Resilience and human adaptability: Who rises above adversity? The American Journal of Occupational Therapy, 45(6), 493-503.

Flach, F. F. (1988). Resilience: Discovering a new strength at times of stress. New York: Fawcett Columbine.

Folkman, S., \& Moskowitz, J. T. (2003). Positive psychology from a coping perspective. Psychological Inquiry, 14(2), 121-125.

Fox, S., \& Spector, P. E. (1999). A model of work frustration—Aggression. Journal of Organizational Behaviour, $20,915-931$.

Fredrickson, B. L., \& Branigan, C. (2005). Positive emotions broaden thought-action repertoires: Evidence for the broaden-and-build model. Cognition and Emotion, 19, 313-332.

Fredrickson, B. L., Tugade, M. M., Waugh, C. E., \& Larkin, G. (2003). What good are positive emotions in crises? A prospective study of resilience and emotions following the terrorist attacks on the United States on September 11, 2001. Journal of Personality and Social Psychology, 84, 365-376.

Greenberg, M. A. (1995). Cognitive processing of traumas: The role of intrusive thoughts and reappraisals. Journal of Applied Social Psychology, 25, 1262-1296.

Hazebroek, J. F., Howells, K., \& Day, A. (2001). Cognitive appraisals associated with high trait anger. Personality and Individual Differences, 30, 31-45.

Horowitz, M. J. (1975). Intrusive and repetitive thoughts after experimental stress: A summary. Archives of General Psychiatry, 32, 1475.

Hunter, A. J., \& Chandler, G. E. (1999). Adolescent resilience. Journal of Nursing Scholarship, 31, 243-247.

Isen, A. M., Daubman, K. A., \& Nowicki, G. P. (1987). Positive affect facilitates creative problem solving. Journal of Personality and Social Psychology, 52, 1122-1131.

Janoff-Bulman, R. (1992). Shattered assumptions: Toward a new psychology of trauma. New York: Free Press.

Litman, J. A., Robert, P. C., \& Spielberger, C. D. (2005). The nature and measurement of sensory curiosity. Personality and Individual Differences, 19, 1123-1133.

MacLeod, C., \& Rutherford, E. M. (1992). Anxiety and the selective processing of emotional information: Mediating roles of awareness, trait and state variables, and personal relevance of stimuli. Behaviour Research and Therapy, 30(5), 479-491.

Manasse, M. E., \& Ganem, N. M. (2009). Victimization as a cause of delinquency: The role of depression and gender. Journal of Criminal Justice, 37(4), 371-378.

Masten, A. S. (2001). Ordinary magic: Resilience processes in development. American Psychologist, 56(3), 227-238.

Michael, S. T., \& Snyder, C. R. (2005). Getting unstuck: The roles of hope, finding meaning, and rumination in the adjustment to bereavement among college students. Death Studies, 29(5), 435-458.

Mogg, K., Bradley, B. P., \& Halowell, N. (1994). Attentional bias to threat: Roles of trait anxiety, stressful events, and awareness. The Quarterly Journal of Experimental Psychology Section A: Human Experimental Psychology, 47(4), 841-864.

Moosbrugger, H., \& Schermelleh-Engel, K. (1991). Determinants of pain experience: Perceived competence, trait anxiety, trait depression and moderator effects. Personality and Individual Differences, 12(12), 1261-1266.

Morrow, J., \& Nolen-Hoeksema, S. (1990). Effects of responses to depression on the remediation of depressive affect. Journal of Personality and Social Psychology, 58(3), 519-527.

Nolen-Hoeksema, S. (1991). Responses to depression and their effects on the duration of depressive episodes. Journal of Abnormal Psychology, 100, 569-582.

Ong, A. D., Bergeman, C. S., Bisconti, T. L., \& Wallace, K. A. (2006). Psychological resilience, positive emotions, and successful adaptation to stress in later life. Journal of Personality and Social Psychology, 91(4), 730-749.

Parrot, D. J., Zeichner, A., \& Evces, M. (2005). Effect of trait anger on cognitive processing of emotional stimuli. Journal of General Psychology, 132(1), 67-80.

Preacher, K. J., \& Hayes, A. F. (2008). Asymptotic and resampling strategies for assessing and comparing indirect effects in multiple mediator models. Behaviour Research Methods, 40(3), 879-891.

Richardson, G. E. (2002). The metatheory of resilience and resiliency. Journal of Clinical Psychology, 58, 307-321.

Roger, D., \& Najarian, B. (1989). The construction and validation of a new scale for measuring emotion control. Personality and Individual Differences, 10(8), 845-853.

Rowe, G., Hirsch, J., \& Anderson, A. K. (2007). Positive affect increases the "breadth" of attentional selection. Proceedings of the National Academy of Sciences of the USA, 104, 338-383.

Rusting, C. L. (1998). Personality, mood, and cognitive processing of emotional information: Three conceptual frameworks. Psychological Bulletin, 124, 165-196. 
Rutter, M. (1985). Resilience in the face of adversity: Protective factors and resistance to psychiatric disorders. British Journal of Psychiatry, 147, 598-611.

Rutter, M. (2007). Resilience, competence, and coping. Child Abuse \& Neglect, 31(3), 205-209.

Salsman, J. M., Segerstrom, S. C., Brechting, E. H., Carlson, C. R., \& Andrykowski, M. A. (2009). Posttraumatic growth and PTSD symptomatology among colorectal cancer survivors: A 3-month longitudinal examination of cognitive processing. Psycho-Oncology, 18, 30-41.

Siegle, G. J., Moore, P. M., \& Thase, M. E. (2004). Rumination: One construct, many features in healthy individuals, depressed individuals, and individuals with lupus. Cognitive Therapy and Research, 28, 645-668.

Spielberger, C. D. (1999) . State-trait anger expression inventory-revised. Odessa, F. L.: Psychological Assessment Resources.

Spielberger, C. D., Jacobs, G. A., Russell, S. F., \& Crane, R. S. (1983). Assessment of anger: The state-trait anger scale. In J. N. Butcher, \& C. D. Spielberger (Eds.), Advances in personality assessment (Vol. 2, pp. 161-189). Hillsdale, N. J.: Lawrence Erlbaum Associates.

Spielberger, C. D., Reheiser, E. C., \& Sydeman, S. J. (1995). Measuring the experience, expression, and control of anger. Issues in Comprehensive Nursing, 18, 207-232.

Spielberger, C. D., \& Sydeman, S. J. (1994). State-trait anxiety inventory and state-trait anger expression inventory. In M. E. Maruish (Ed.), The use of psychological tests for treatment planning and outcome assessment (pp. 292-321). Hillsdale, N. J.: Lawrence Erlbaum Associates.

Tafrate, R. C., Kassinove, H., \& Dundin, L. (2002). Anger episodes in high- and low-trait-anger community adults. Journal of Clinical Psychology, 59, 1573-1590.

Tedeschi, R. G., \& Calhoun, L. G. (2004). Post-traumatic growth: Conceptual foundations and empirical evidence. Psychological Inquiry, 15, 1-18.

Tugade, M. M., \& Fredrickson, B. L. (2004). Resilient individuals use positive emotions to bounce back from negative emotional experiences. Journal of Personality and Social Psychology, 86, 320-333.

Van Honk, J., Tuiten, A., de Haan, E., van den Hout, M., \& Stam, H. (2001). Attentional biases for angry faces: Relationships to trait anger and anxiety. Cognition and Emotion, 15, 279-299.

Wilkowski, B. M., \& Robinson, M. D. (2008). The cognitive basis of trait anger and reactive aggression: An integrative analysis. Personality and Social Psychology Review, 12, 3-21. 